\title{
REVIEW \\ Protective effects of nebivolol from oxidative stress to prevent hypertension-related target organ damage
}

\author{
A Coats ${ }^{1}$ and S Jain ${ }^{2}$
}

\begin{abstract}
Hypertension is one of the leading risk factors for morbidity and mortality in patients with cardiovascular and cerebrovascular diseases and renal impairment. It also leads to target organ damage (TOD), which worsens organ function and the patient's clinical status. Reactive oxygen species (ROS)-mediated oxidative stress may contribute significantly to TOD in patients with hypertension. NO (nitric oxide) is a paracrine factor derived from endothelial cells that has been shown to alleviate ROS-mediated oxidative damage. Nebivolol is a third-generation $\beta$-blocker with vasodilator activity, both actions contributing to decreased blood pressure in hypertensive patients. Its vasodilatory function is mediated by the endothelial L-arginine NO pathway. Nebivolol increases the bioavailability of NO in the vasculature. Its efficacy and safety profile is comparable to other commonly used antihypertensive agents. In this article, we review the current literature to understand TOD secondary to oxidative stress in patients with hypertension and the role of nebivolol in its prevention. A better understanding of the underlying mechanisms by which nebivolol reduces ROS-mediated TOD will not only help in the development of targeted therapies but may also improve health outcomes in hypertensive patients.
\end{abstract}

Journal of Human Hypertension (2017) 31, 376-381; doi:10.1038/jhh.2017.8; published online 2 March 2017

\section{INTRODUCTION}

Target organ damage (TOD) is one of the most feared consequences of uncontrolled hypertension. The presence of TOD leads to an increase in cardiovascular mortality and morbidity. ${ }^{1}$ Primarily the heart, kidney, brain and the vasculature are affected by the damage that results from elevated blood pressure. Established TOD may lead to coronary heart disease, myocardial infarction, heart failure, ischaemic and haemorrhagic stroke, and atherosclerotic changes (development of stenosis, aneurysm) and renal failure. ${ }^{2}$ According to The European Society of Hypertension (ESH), evaluation of subclinical organ damage at an early stage is crucial in determining cardiac risk in patients with hypertension. ${ }^{3}$ Oxidative stress is one of the most important mechanisms implicated in TOD in patients with hypertension. ${ }^{4}$ It is defined as an increase in vascular reactive oxygen species (ROS), which in turn decreases the level of antioxidant activity in the vasculature. $^{4}$ Nicotinamide adenine dinucleotide phosphate oxidase, xanthine oxidase and uncoupled nitric oxide synthase (NOS) are among the major sources of ROS in vascular disease and hypertension. ${ }^{5}$ TOD, such as left ventricular hypertrophy, carotid atheromatosis and renal impairment, is considered as an intermediate stage in the continuum of vascular disease. ${ }^{3} \mathrm{~A}$ study including 1095 consecutive hypertensive subjects showed a prevalence of TOD in $44.5 \%$ of the hypertensive population. ${ }^{1}$ Segura et $a .^{6}{ }^{6}$ reported the presence of at least one manifest TOD in $61.3 \%$ of the hypertensive $(n=5243)$ and $30.8 \%(n=308)$ of the pre-hypertensive patients. Hence, the assessment and management of TOD in hypertensive patients is important and should be taken into account when planning clinical care which may improve their cardiovascular prognosis. ${ }^{3}$
Hypertensive patients with a known history of diabetes mellitus, cardiovascular, cerebrovascular and/or renal disease are high risk patients. ${ }^{3}$ According to the ESH guidelines, target blood pressure should be $<140 / 90$ for low-moderate risk hypertensives and $<130 / 80 \mathrm{~mm} \mathrm{Hg}$ for high-risk patients. ${ }^{3} \mathrm{~A}$ decrease in blood pressure by $20 / 10 \mathrm{~mm} \mathrm{Hg}$ results in a decrease in mortality in cerebrovascular, cardiovascular and chronic kidney disease patients. $^{7}$

Nebivolol is a third-generation $\beta$-blocker. A direct relationship between oxidative stress and TOD via the NO pathway exists in the patients with hypertension. Thus, nebivolol mediated increase in bioavailability of NO in vasculature may provide added benefits in reducing TOD in patients with hypertension. Therefore, we intend to discuss the mechanism by which oxidative stress leads to TOD and the role of nebivolol in preventing the same in this review.

\section{NEBIVOLOL: A CARDIO-SELECTIVE $\boldsymbol{\beta}$-BLOCKER}

It is recommended at a standard oral dose of $5 \mathrm{mg}$ for the treatment of hypertension. It reaches its peak plasma level within $2 \mathrm{~h}$. In the elderly (>65 years) its starting dose has been recommended as $2.5 \mathrm{mg}$ per day. In poor CYP2D6 metabolizers, its bioavailability is very high (96\%); however, in extensive metabolizers, it is about 12\%; although no significant difference has been found between the two profiles in terms of safety and efficacy. ${ }^{8}$ Several randomized, double-blind, placebo-controlled clinical trials have assessed the efficacy and safety of nebivolol in patients with hypertension. ${ }^{8}$ The overall response rate (decrease in sitting/supine diastolic blood pressure to $\leqslant 90 \mathrm{~mm} \mathrm{Hg}$ or a $10 \%$ or $10 \mathrm{~mm} \mathrm{Hg}$ fall in diastolic blood) to nebivolol therapy has been

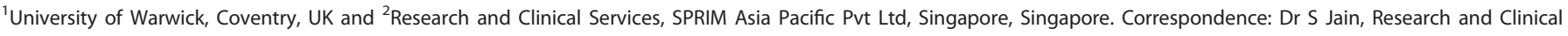
Services, SPRIM Asia Pacific Pvt Ltd, 6 Rochester Park, Singapore 139217, Singapore. 
shown to be in the range of $58-81 \%$ after $4-52$ weeks of treatment. ${ }^{8}$ In a clinical trial, the overall response rate, defined as normalization of or $>10 \%$ reduction in diastolic blood pressure, was $65 \%$ in patients treated with nebivolol (5 mg, daily) as compared with $25 \%$ in placebo after 4 weeks of treatment. ${ }^{9}$ Nebivolol significantly reduced blood pressure as soon as 15 days after the start of treatment. ${ }^{10}$ In a large study with 6356 mild hypertensive patients, nebivolol therapy for 6 months resulted in significantly reduced blood pressure with similar efficacy of nebivolol monotherapy and add-on therapy. ${ }^{11}$ Another study that focused on the long-term efficacy of nebivolol monotherapy found a significant reduction in blood pressure after 6 months of treatment. ${ }^{10}$

Nebivolol $5 \mathrm{mg}$ once daily was well tolerated with no signs of orthostatic hypotension. ${ }^{8}$ A meta-analysis of 12 clinical trials revealed that significantly fewer patients treated with nebivolol reported adverse events as compared with other antihypertensive drugs (OR 0.59; $P=0.007$ ). ${ }^{12}$ However, nebivolol's adverse effects tended to be transient and of mild to moderate intensity. ${ }^{8}$ The most common adverse effects (reported by $<10 \%$ of patients) are headache, dizziness, paraesthesia, dyspnoea, constipation, nausea, diarrhoea, tiredness and oedema. ${ }^{8}$ Notably, it has not shown any adverse effect on glucose and lipid metabolism. ${ }^{13}$ Based on a self-administered questionnaire containing 47 questions regarding common complaints, it was observed that nebivolol does not impair quality of life in patients with hypertension as assessed by the ISH (inventory to subjective health). ${ }^{9}$ Additionally, nebivolol has several benefits as compared with other $\beta$-blockers, such as improvement in sleep parameters and a lower incidence of erectile dysfunction, possibly due to increased NO availability. ${ }^{14,15}$ Nebivolol improves exercise capacity in patients with hypertension and heart failure to similar extent as other antihypertensive drugs. ${ }^{15}$ Nebivolol also exhibits significant vasodilatory activity via the L-arginine/NO pathway and increases its bioavailability in the vasculature. $^{14}$

\section{ROLE OF NEBIVOLOL IN REDUCING OXIDATIVE DAMAGE}

Oxidative damage

A common feature of many conditions associated with vasoconstriction is vascular oxidative stress. An imbalance between the generation of ROS and the antioxidant defence systems causes oxidative stress. The reactive species include superoxide $\left(\mathrm{O}_{2}^{-}\right)$, hydroxyl radical $(\mathrm{HO} \cdot)$, hydrogen peroxide $\left(\mathrm{H}_{2} \mathrm{O}_{2}\right)$, peroxynitrite $\left(\mathrm{ONOO}^{-}\right)$, nitrogen oxide $(\mathrm{NO} \bullet)$ and hypochlorous acid $(\mathrm{HOCl})$. Oxidative stress promotes vascular smooth muscle cell proliferation, hypertrophy and collagen deposition, leading to thickening of the vascular media and narrowing of the vascular lumen. In addition, increased oxidative stress also damages the endothelium, impairs endothelium-dependent vascular relaxation and increases vascular contractile activity. Oxygen radicals may also induce endothelial permeability, with extravasation of plasma proteins and other macromolecules and recruitment of inflammatory proteins and cells. These could further impair endothelial function and aggravate vascular damage. ${ }^{4}$

NO is recognized as a ubiquitous intercellular messenger modulating crucial functions, including blood flow, platelet aggregation and neuronal activity and has an important role in the modulation of vascular tone. Endothelium-derived NO is a key regulator of endothelial function. ${ }^{5}$ The NO pathway is directly implicated in the development and progression of several diseases and is considered a promising target for developing new clinical strategies against cardiovascular pathologies. ${ }^{5}$ It is generated by endothelial NOS (eNOS). Researchers have demonstrated that the loss of $\mathrm{NO}$ is one of the earliest events that lead to the onset and progression of oxidative stress particularly in the vasculature. Decreased production or increased destruction of
NO leads to endothelial dysfunction. Additionally, an increased production and/or impaired inactivation of ROS renders eNOS dysfunctional, which may also lead to reduced bioactivity of NO. The resultant NO deficiency creates an environment that is conducive to vascular disorders including hypertension. Adrenergic vasoconstriction is a balance between a direct vasoconstrictive effect on smooth muscle and an indirect vasorelaxant action caused by a2- and $\beta$-adrenergic endothelial receptor-triggered NO release. An increased oxidative stress due to reduction of NO bioavailability shifts this equilibrium. This shift results in enhanced vascular adrenergic responsiveness which is observed in hypertension. Oxidative stress plays a significant role in the development of hypertension, in part by inactivation of $\mathrm{NO}$ in vivo. ${ }^{5}$ Substantial evidence from animal and human studies exists to support the role of $\mathrm{NO}$ in reducing oxidative stress and modulating blood pressure in literature.

The role of nebivolol in reducing oxidative damage

Nebivolol has emerged as a preferred antihypertensive agent due to its dual properties of $\beta$-blockade and vasodilation (via NO pathway). It is a racemic mixture (1:1) of two enantiomers, L-nebivolol and D-nebivolol. Both isomers provide vasodilating effect, with the vasodialating effect being more marked for L-nebivolol while the D-isomer provides practically all the $\beta$-blocking activity. Apart from its $\beta$-adrenoreceptor blockade, vasodilation through the NO pathway contributes significantly to the reduction of blood pressure by nebivolol. Vasodilation associated with nebivolol is due to its effects on the L-arginine/ NO pathway in the endothelium of blood vessels. Nebivolol stimulates $\beta_{3}$-adrenergic receptors from endothelial cells which results in the activation of eNOS and NO release. Release of NO via the endothelium causes peripheral vasodilation, increased myocardial compliance and inhibition of the inotropic effects of excessive sympathetic stimulation. Indirectly, nebivolol also reduces the NO degradation and increases the bioavailability of $\mathrm{NO}^{13}$ All those NO-mediated effects may be beneficial leading to a reduced cardiac load, improved cardiac filling and protection of the myocardium from excessive sympathetic stimulation. ${ }^{15}$

As compared with conventional $\beta$-blockers, nebivolol is highly selective for cardiac $\beta 1-A R s$ and display the highest $\beta 1 / \beta 2$ selectivity compared with other $\beta$-blockers, with no appreciable effect on a-receptors. It has shown a better haemodynamic and safety profile along with additional vascular protection as compared with conventional $\beta$-blockers. In a meta-analysis of 12 clinical trials, it was found that patients treated with nebivolol displayed a higher antihypertensive response rate (OR 1.44; $P=0.001$ ) as compared with the other antihypertensive drugs. ${ }^{12}$ Nebivolol significantly reduced heart rate and peripheral resistance and increased stroke volume, leading to a small increase in cardiac output as compared with atenolol. ${ }^{16}$ Improvements in diastolic function also highlighted its potential for use in the treatment of heart failure. In a double-blind, randomized design, 20 healthy subjects and 20 matched essential hypertensive patients were treated with atenolol or nebivolol. ${ }^{17}$ There was a greater reduction of ROS concentration in endothelial cells exposed to oxidative stress in endothelial cells of the patients receiving nebivolol compared with the atenolol group. Furthermore, the reduction of basal and stimulated NO induced by oxidative stress in endothelial cells was significantly lower in the patients receiving nebivolol. Thus, nebivolol displays significant antioxidant properties which may help in reducing oxidative stress-mediated organ damage in the patients with hypertension. 


\section{OXIDATIVE DAMAGE TO CARDIOVASCULAR SYSTEM: A ROLE FOR NEBIVOLOL}

Oxidative damage to cardiovascular system

NO bioavailability plays an important role in the pathophysiology of cardiovascular disease (CVD). A decrease in the NO level in endothelial cells is associated with endothelial dysfunction and hypertension. The association between endothelial dysfunction and vascular diseases is well characterized. Cardiovascular homoeostasis is regulated by NO produced by all three NOS isoforms; neuronal NOS, eNOS and inducible NO synthase. Several studies have demonstrated that NO produced by neuronal NOS controls catecholamine's release in response to electrical adrenergic nerve stimulation, both in vivo and in vitro. In addition, elevated levels of catecholamines are also associated with several pathologic conditions such as heart failure. In the heart and vessels, endothelial cells express eNOS, which is responsible for the production of NO in response to stimulation of adrenergic and muscarinic cholinergic receptors in cardiac myocytes. The effect of acute and chronic $\beta$-adrenergic activation on endothelial NO synthase activity, expression and uncoupling is depicted in Figure 1. The main mechanism leading to increased eNOS activity in endothelial cells is calcium-dependent, but phosphorylation at several loci of the NOS proteins has also been recognized as an additional pathway to induce both activation and inhibition of eNOS activity. Many studies have demonstrated that vascular endothelial cells also express $\beta$-adrenoceptors, thus supporting the hypothesis that the endothelium might control or facilitate $\beta$-adrenergic effects on the vessels. The inducible NO synthase is involved in several aspects of cardiovascular biology including the defense against intracellular microorganisms. The activity of NOS contributes to the homoeostasis of the adrenergic pathway, thus supporting the hypothesis that the endothelium might control or facilitate $\beta$-adrenergic effects on the vessels and the polymorphic variants in $\beta 3$-receptors and NOS isoforms could influence aging, few pathological conditions and individual responses to drugs. This seems to be dependent, almost in part, on differences in the control of vascular tone exerted by NO. Given its involvement in such important mechanisms, the NO pathway is implicated in the aging process as well as in cardiovascular and non-cardiovascular conditions. Thus, it is essential to pinpoint involvement of $\mathrm{NO}$ in the regulation of vascular tone for effective management of CVDs. Furthermore, oxidative stress due to endothelial dysfunction has also been implicated in the development of atherosclerosis in hypertensive patients, which is mediated by nicotinamide adenine dinucleotide phosphate oxidase-derived ROS. $^{4,5}$

The role of nebivolol in reducing oxidative damage to the cardiovascular system

Although several third-generation $\beta$-blockers have demonstrated vasodilating properties, only nebivolol has shown to mediate a vasodilatory effect via NO release, providing a unique dual mechanism of action, which clearly differentiates it from other $\beta$-blockers such as atenolol or carvedilol. NO release plays an important role in endothelial protection and may provide benefits beyond blood pressure reduction in a variety of cardiovascular conditions. ${ }^{12}$ The favourable haemodynamic profile of nebivolol, including the lowering of blood pressure, is partially due to NO release from endothelial cells. ${ }^{18}$ In addition, due to its antioxidant activity, nebivolol prevents the detrimental effect on NO bioavailability associated with oxidative stress. ${ }^{5}$ Stimulation of endothelial $\beta$-adrenoreceptors improves eNOS-derived NO production. Nebivolol has a distinctive profile among $\beta$-blockers, with the greatest selectivity for cardiac $\beta 1$-ARs and the highest $\beta 1 / \beta 2$ selectivity as compared with other $\beta$-blockers. ${ }^{5}$ Additionally, nebivolol enhances NO release and may promote neo-angiogenesis in cardiac tissue via stimulation of $\beta 3$-ARs. ${ }^{5}$ Hence, it reduces heart rate and blood pressure and improves systolic and diastolic function. ${ }^{5}$ It has been demonstrated that permanent $\beta$-ARs stimulation, typically observed during CVD, could induce an overexpression and activation of eNOS, which in turn could lead to oxidative stress through superoxide anion generation and paradoxically a consequent decrease of NO bioavailability. ${ }^{5}$ Furthermore, in vitro NO-mediated platelet aggregation and smooth muscle cell proliferation were observed to be significantly increased by nebivolol, which may contribute to the cardio-protective effects of nebivolol in myocardial ischaemia. $^{13}$
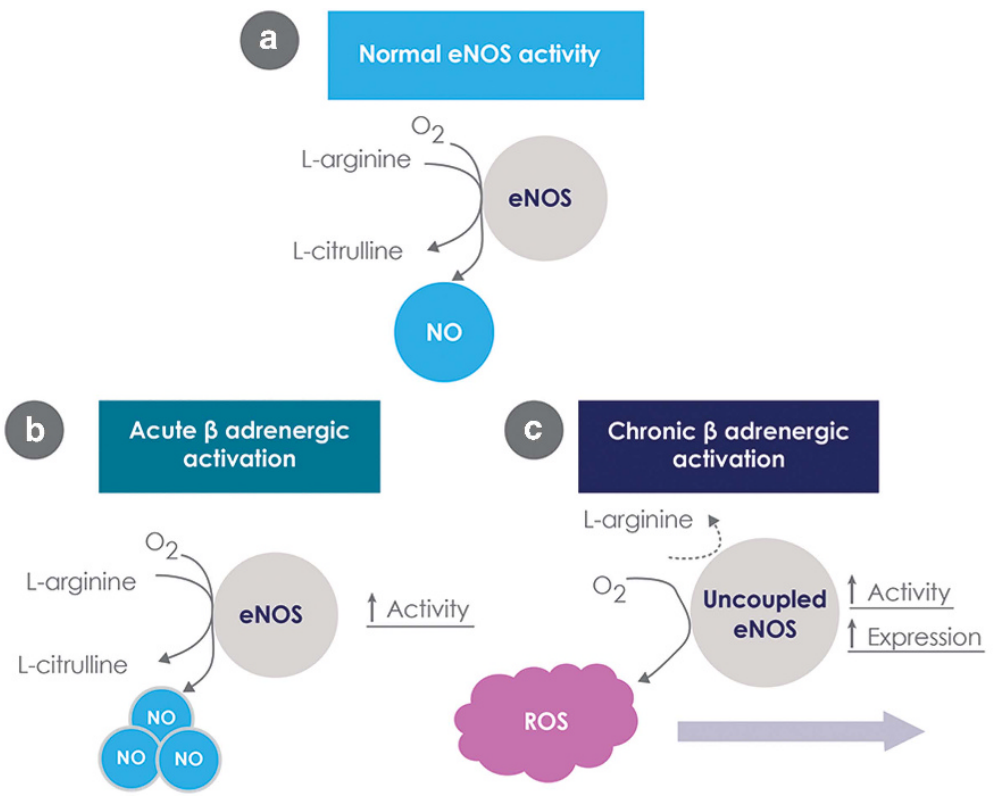

Figure 1. eNOS activity, expression and uncoupling in (a) normal conditions, (b) acute $\beta$-adrenergic activation and (c) chronic $\beta$-adrenergic activation. eNOS, endothelial nitric oxide synthase; NO, nitric oxide; ROS, reactive oxygen species. ${ }^{15}$ 


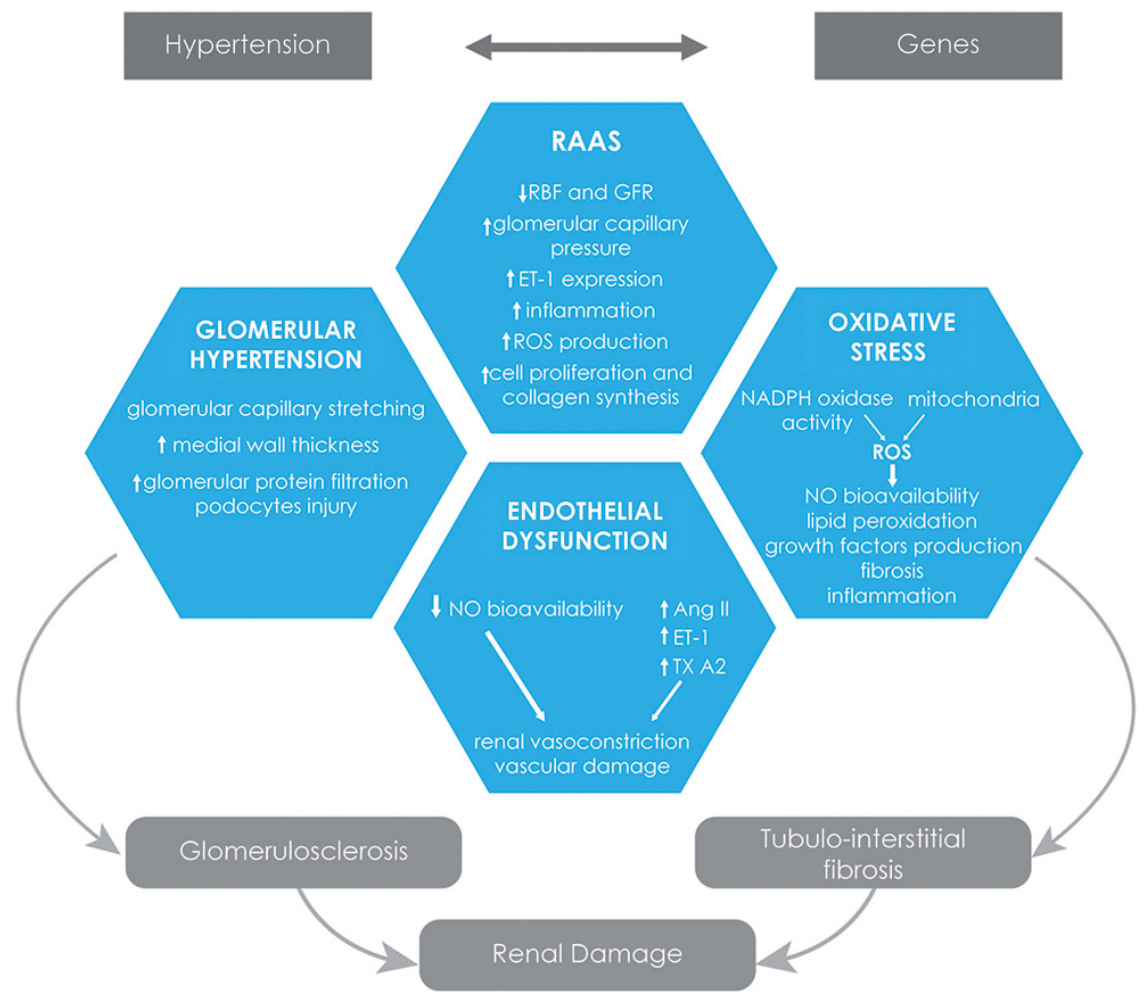

Figure 2. Mechanisms connecting hypertension and renal nephropathy. ET-1, endothelin-1; GFR, glomerular filtration rate; NADPH, nicotinamide adenine dinucleotide phosphate; NO, nitric oxide; RBF, renal blood flow; ROS, reactive oxygen species; TX A2, thromboxane A2. ${ }^{2}$

Numerous clinical studies highlight the role of NO in nebivolol's mechanism of action. The ENECA clinical trial reported a significant increase in left ventricular ejection fraction in nebivolol-treated elderly patients (>65 years) with chronic heart failure as compared with placebo. ${ }^{19}$ The SENIORS study has also shown that over a mean period of 21 months, significantly fewer $(\mathrm{HR}=0.86, \quad \mathrm{Cl} \quad 0.74-0.99 ; \quad P=0.039)$ elderly patients ( $>70$ years) reached the primary end point of all-cause mortality or cardiovascular hospital admission (time to first event) as compared with placebo. ${ }^{20}$ It has been suggested that nebivolol may reduce the risk of atherosclerotic plaque rupture by decreasing the blood pressure on the arterial walls and inhibiting thrombus formation after plaque rupture. ${ }^{12}$ Furthermore, a sub-analysis of SENIORS trial data revealed that nebivolol reduced cardiac ischaemic events in patients with heart failure of ischaemic aetiology in 2128 elderly heart failure patients of more than 70 years of age with coronary artery disease. ${ }^{21}$ Interestingly, the authors mentioned pharmacological preconditioning as one of the underlying mechanisms for the protective effects of nebivolol in heart failure patients with coronary artery disease along with above-mentioned NOmediated effects, which translate into coronary vasodilation, reduced platelet aggregation and possible induction of nitratelike preconditioning.

Nebivolol has also been shown to decrease QT dispersion and corrected QT interval (QTC) and QT dispersion (QTcd), which are indicative of myocardial repolarization and electrical instability in heart failure patients with systolic dysfunction. ${ }^{22}$ In a clinical trial conducted among 16 patients with stable angina of effort, nebivolol increased ischaemic and angina thresholds for at least $8 \mathrm{~h}$ as compared with placebo. ${ }^{23}$ As compared with atenolol, nebivolol has shown a significant increase in flow- mediated dilatation in patients with stable coronary artery disease. $^{18}$

Nebivolol improved small artery distensibility, which is a measure of arterial compliance or stiffness, and is considered to be an independent predictor of mortality in patients with essential hypertension. ${ }^{14}$ In addition, nebivolol also decreases markers of arterial stiffness, including central aortic pressures, augmentation index and carotid femoral pulse which are superior indicators of CVD. ${ }^{24}$ Nebivolol may have an effect on small resistance arteries, by increasing pulse pressure amplification and reducing wave reflection owing to its property to increase NO bioavailibility. ${ }^{25}$ Nebivolol induced a potent vasodilation in the forearm, which was hampered by NG-monomethyl L-arginine, a strong NO inhibitor. ${ }^{5}$ Hence, the antioxidative property of nebivolol makes it an attractive therapeutic option for various other cardiovascular conditions besides hypertension. In summary, nebivolol has been shown to reduce blood pressure and heart rate, peripheral resistance, left ventricular mass, reflected wave and central aortic pulse pressures. ${ }^{13,24}$ It increases left ventricular ejection fraction (i.e. improved systolic function), E/A ratio (improved diastolic function) and stroke volume with the preservation of cardiac output. $^{13}$ It also improves coronary flow reserve, ventricular function, diastolic function and corrects endothelial dysfunction. ${ }^{8}$

\section{OXIDATIVE DAMAGE TO THE KIDNEY: A ROLE FOR NEBIVOLOL}

Oxidative damage to kidney

Microalbuminuria and reduced glomerular filtration rate can be found in the early stages of hypertension and result in chronic renal failure in the long run. ${ }^{26}$ Renal impairment including renal 
arteriolar thickening, fibrinoid deposition and proteinuria is often found in patients with hypertension. Chronic hypertension may lead to nephroangiosclerosis. In addition, elevated blood pressure has been considered as an independent risk factor for impaired renal function and end-stage renal disease. ${ }^{26}$ Oxidative stress is one of the major underlying mechanism for renal damage in hypertensive patients as depicted in Figure $2 .^{27}$

ROS are produced in arterioles, glomerular and tubular cells, the macula densa and podocytes in the kidney. Moreover, vasoactive agents, inflammation, mechanical factors (shear stress) and metabolic factors also contribute to ROS production through nicotinamide adenine dinucleotide phosphate or mitochondria, which results in decreased NO bioavailability. ROS may also increase afferent arteriolar tone and reactivity via potentiation of tubuloglomerular feedback and microvascular mechanisms that diminish endothelium-derived relaxation factor/NO responses. This may generate a cyclooxygenase-2-dependent endothelialderived contracting factor that activates thromboxane-prostanoid receptors, and enhances vascular smooth muscle cells reactivity. ROS also reduce $\mathrm{pO}_{2}$ in the kidney cortex which results in decreased efficiency of the kidney to use $\mathrm{O}_{2}$ for $\mathrm{Na}^{+}$transport which may induce vascular damage and hypertension. It has been found that uric acid also plays a role in hypertension via stimulation of oxidative stress, endothelial dysfunction, inflammation and vasoconstriction, and contribute to the pathogenesis of hypertensive nephropathy. ${ }^{27}$ According to ESH/ESC 2013 guidelines, all hypertensive patients should undergo testing for microalbuminuria and an estimation of glomerular filtration rate should be made. ${ }^{3}$

The role of nebivolol in reducing oxidative damage to the kidney Nebivolol reduces kidney perfusion pressure, which resulted in increased renal plasma flow and GFR in a dose dependent and coherent manner in the experimental model. ${ }^{18}$ Increased GFR also increases urine flow and excretion of sodium and chloride. Longterm use of nebivolol reduces renal fibrosis in rats via a mechanism independent of its $\beta$-blocking activity. ${ }^{18}$ Another study reported a significant nephroprotective effect of nebivolol in a rat model of renal failure as measured by increased albuminuria and acetylcholine relaxation. ${ }^{28}$

At recommended doses, nebivolol decreased plasma renin (by $52 \%, P=0.001$ ) and aldosterone levels (by $28 \%$ in 2 weeks) in hypertensive and normotensive patients. ${ }^{29}$ Atrial natriuretic peptide (a vasorelaxant) is also found to be elevated in plasma by $60 \%$ in 2 weeks in patients with hypertension. ${ }^{29}$ In the SENIORS trial, no major safety issue was found in elderly chronic heart failure patients with moderate renal impairment (estimated glomerular filtration rate $<60$ ) using nebivolol. ${ }^{20}$ In a pilot study of 33 patients with severe renal artery stenosis who underwent revascularization, nebivolol $(n=17)$ significantly increased estimated glomerular filtration rate and decreased proteinuria as compared with the control group. ${ }^{30}$ Nebivolol was also found to be effective in nephrotoxicity induced by contrast media. ${ }^{31}$ In patients pre-treated with nebivolol before coronary angiography and ventriculography, it was found that nebivolol did not increase serum creatinine. Taken together, these evidence suggest that nebivolol may have a nephroprotective effect in hypertensive patients, and helps in preventing renal damage due to oxidative stress. The most recent ESC heart failure treatment guidelines of 2016 stated that in older patients with heart failure, nebivolol reduced the combined end point of death or cardiovascular hospitalization with no significant interaction between treatment effect and baseline left ventricular ejection fraction. ${ }^{32}$

\section{OXIDATIVE DAMAGE TO THE BRAIN: A ROLE FOR NEBIVOLOL}

Oxidative damage to brain

High blood pressure is one of the most important risk factor for stroke. Small vessel disease, such as silent lacunar infarcts, advanced deep white matter lesions and microbleeding, may lead to ischaemic and haemorrhagic stroke and vascular dementia in patients with hypertension. ${ }^{33}$ In hypertensive patients, magnetic resonance imaging revealed the presence of silent cerebrovascular lesions in $44 \%$ of the patients, much more than cardiac (21\%) and renal $(26 \%)$ subclinical damage. ${ }^{3}$ It has been suggested that microcirculation disorders and endothelial dysfunction may be a reason for impaired cognitive function in hypertensive patients. ${ }^{34}$ Hypoperfusion of the brain due to arterial stiffness has been reported in hypertensive patients. $^{35}$ Hypertension-induced lipohyalinosis and fibrinoid degeneration has also been found in white matter hyperintensities. ${ }^{33}$

Role of nebivolol in reducing oxidative damage to brain Arterial hypertension may lead to brain damage. ${ }^{35}$ Oxidative stress, inflammation and abnormalities in blood-brain barrier have been found to be underlying mechanisms for brain damage. ${ }^{35}$ In addition, it has been reported that the brain has reduced antioxidant defences. ${ }^{36}$ Antihypertensive drugs have been shown to reduce the risk of stroke and other cerebrovascular disease and the accompanying brain damage. ${ }^{3,26}$ Active antihypertensive treatment significantly reduces vascular dementia in older patients with isolated systolic hypertension. ${ }^{33,34}$ As nebivolol possesses significant antioxidant activity, its role in preventing cerebrovascular damage owing to ROS and inflammation may be postulated. In addition, nebivolol has also been shown to reduce arterial stiffness, which is a leading cause of vascular damage in the brain. ${ }^{25}$ However, systematic in vivo and clinical studies are required to investigate the role of nebivolol in preventing oxidative stress and inflammation-mediated brain damage in hypertensive patients.

\section{CONCLUSION/PERSPECTIVE}

Subclinical and clinical organ damage in the cardiovascular (left ventricular hypertrophy, atrial fibrillation, heart failure, coronary events, increased arterial stiffness and atherosclerotic changes), renal (proteinuria and renal failure) and cerebrovascular systems remain the major cause of death among patients with hypertension. Hence, appropriate management of high blood pressure is crucial to reduce mortality and morbidity in such patients. The heterogeneous nature of the underlying mechanisms for hypertension and the availability of more than 75 drugs in nine different pharmacologic groups add to the complexity in its management. It is important to understand various genetic (gender, ethnicity, race) factors, environmental factors, and the role of the renal artery stenosis/adrenergic system, salt/water retention, arterial stiffness, presence of TOD in the development of effective drug therapy for hypertensive patients. Nebivolol is a promising antihypertensive drug and demonstrates strong antioxidative properties due to its NO-releasing capabilities. It has shown considerable efficacy and safety in reducing blood pressure and preventing organ damage, and its role as an effective disease modifying agent in elderly heart failure patients irrespective of left ventricular ejection fraction, making it an attractive option in the treatment of high-risk hypertension. Its complete therapeutic profile needs to be further evaluated including its protective effects on TOD and death in large outcome-based clinical trials. Additional randomized controlled trials comparing effects of nebivolol with other commonly used antihypertensive drugs on TOD and death are also warranted. Results from such clinical studies will immensely help in designing therapeutic strategies for 
the management of hypertension and in the prevention of associated TOD and death in hypertensive patients.

What is known about topic?

- Hypertension leads to target organ damage (TOD) in cardiovascular, renal, and cerebrovascular systems

- Reactive oxygen species (ROS) mediated oxidative stress significantly contribute to TOD

- Nebivolol, a third generation $\beta$-blocker has significant anti-oxidative activity through L-arginine/NO pathway

What this study adds?

- Evaluation of underlying mechanisms by which nebivolol reduces ROS-mediated target organ damage

- Summary of available clinical evidence for anti-oxidative properties of nebivolol

\section{CONFLICT OF INTEREST}

This publication was sponsored by A Menarini Asia Pacific Pte Ltd. Clinical authors have received an honorarium for the development and manuscript writing.

\section{ACKNOWLEDGEMENTS}

Editorial support for the preparation of this manuscript was given by SPRIM Asia Pacific Pvt Ltd, Singapore through an unrestricted educational grant by A Menarini Asia Pacific Pte. Ltd.

\section{REFERENCES}

1 Papazafiropoulou A, Skliros E, Sotiropoulos A, Papafragos C, Gikas A, Apostolou O et al. Prevalence of target organ damage in hypertensive subjects attending primary care: C.V.P.C. study (epidemiological cardio-vascular study in primary care). BMC Fam Pract 2011; 12: 75.

2 Schmieder RE. End organ damage in hypertension. Dtsch Arztebl Int 2010; 107: 866-873.

3 Mancia G, Fagard R, Narkiewicz K, Redón J, Zanchetti A, Böhm M et al. 2013 ESH/ ESC Guidelines for the Management of Arterial Hypertension. J Hypertens 2013; 31: 1281-1357.

4 Touyz RM, Briones AM. Reactive oxygen species and vascular biology: implications in human hypertension. Hypertens Res 2011; 34: 5-14.

5 Conti V, Russomanno G, Corbi G, Izzo V, Vecchione C, Filippelli A. Adrenoreceptors and nitric oxide in the cardiovascular system. Front Physiol 2013; 4: 321.

6 Segura J, De La Sierra A, Fernandez S, Ruilope LM. High prevalence of target organ damage in hypertensive and prehypertensive patients with associated cardiovascular risk factors. J Hypertens 2010; 28 (e-Supplement A):26.62.

7 National Clinical Guideline Centre UK. The Clinical Management of Primary Hypertension in Adults. The National Clinical Guideline Centre at The Royal College of Physicians: London, UK, 2011.

8 McNeely W, Goa KL. Nebivolol in the management of essential hypertension: a review. Drugs 1999; 57: 633-651.

9 Van Bortel LM, Breed JG, Joosten J, Kragten JA, Lustermans FA, Mooij JM. Nebivolol in hypertension: a double-blind placebo-controlled multicenter study assessing its antihypertensive efficacy and impact on quality of life. $J$ Cardiovasc Pharmacol 1993; 21: 856-862.

10 Cleophas TJ, Grabowsky I, Niemeyer MG, Mäkel WN, van der Wall EE. Long-term efficacy of nebivolol monotherapy in patients with hypertension. Curr Ther Res 2001; 62: 451-461.

11 Cleophas TJ, Agrawal R, Lichtenthal A, Mäkel W, Fici F. Nationwide efficacy-safety study of nebivolol in mildly hypertensive patients. Am J Ther 2006; 13: 192-197.

12 Van Bortel LM, Fici F, Mascagni F. Efficacy and tolerability of nebivolol compared with other antihypertensive drugs: a meta-analysis. Am J Cardiovasc Drugs 2008; 8: 35-44.

13 Moen MD, Wagstaff AJ. Nebivolol: a review of its use in the management of hypertension and chronic heart failure. Drugs 2006; 66: 1389-1409; discussion 1410.

14 Weiss R. Nebivolol: a novel beta-blocker with nitric oxide-induced vasodilatation. Vasc Health Risk Manag 2006; 2: 303-308.

15 Toblli JE, DiGennaro F, Giani JF, Dominici FP. Nebivolol: impact on cardiac and endothelial function and clinical utility. Vasc Health Risk Manag 2012; 8: 151-160.
16 Kamp O, Sieswerda GT, Visser CA. Comparison of effects on systolic and diastolic left ventricular function of nebivolol versus atenolol in patients with uncomplicated essential hypertension. Am J Cardiol 2003; 92: 344-348.

17 Fratta Pasini A, Garbin U, Nava MC, Stranieri C, Davoli A, Sawamura T et al. Nebivolol decreases oxidative stress in essential hypertensive patients and increases nitric oxide by reducing its oxidative inactivation. J Hypertens 2005; 23: 589-596.

18 Maffei A, Lembo G. Nitric oxide mechanisms of nebivolol. Ther Adv Cardiovasc Dis 2009; 3: 317-327.

19 Edes I, Gasior Z, Wita K. Effects of nebivolol on left ventricular function in elderly patients with chronic heart failure: results of the ENECA study. Eur J Heart Fail 2005; 7: 631-639.

20 Cohen-Solal A, Kotecha D, van Veldhuisen DJ, Babalis D, Böhm M, Coats AJ et al. Efficacy and safety of nebivolol in elderly heart failure patients with impaired renal function: insights from the SENIORS trial. Eur J Heart Fail 2009; 11: 872-880.

21 Ambrosio G, Flather MD, Bohm M, Cohen-Solal A, Murrone A, Mascagni F et al. Beta-blockade with nebivolol for prevention of acute ischaemic events in elderly patients with heart failure. Heart 2011; 97: 209-214.

22 Aksoy SM, Cay S, Cagirci G, Sen N. Nebivolol therapy improves QTC and QTcd parameters in heart failure patients. Cardiovasc J Afr 2012; 23: 191-193.

23 Cherchi A, Lai C, Pirisi R, Onnis E. Antianginal and anti-ischaemic activity of nebivolol in stable angina of effort. Drug Investig 1991; 3(Suppl 1): 86-96.

24 Soanker R, Naidu MUR, Raju SB, Prasad AK, Rao TRK. Effect of beta-1-blocker, nebivolol, on central aortic pressure and arterial stiffness in patients with essential hypertension. Indian J Pharmacol 2012; 44: 407-411.

25 Agabiti-Rosei E, Porteri E, Rizzoni D. Arterial stiffness, hypertension, and rational use of nebivolol. Vasc Health Risk Manag 2009; 5: 353-360.

26 Persu A, De Plaen J-F. Recent insights in the development of organ damage caused by hypertension. Acta Cardiol 2004; 59: 369-381.

27 Mennuni S, Rubattu S, Pierelli G, Tocci G, Fofi C, Volpe M. Hypertension and kidneys: unraveling complex molecular mechanisms underlying hypertensive renal damage. J Hum Hypertens 2014; 28: 74-79.

28 Gschwend S, Haug MB, Nierhaus M, Schulz A, Vetter R, Kossmehl P et al. Shortterm treatment with a beta-blocker with vasodilative capacities improves intrarenal endothelial function in experimental renal failure. Life Sci 2009; 85: 431-437.

29 Chan TY, Woo KS, Nicholls MG. The application of nebivolol in essential hypertension: a double-blind, randomized, placebo-controlled study. Int J Cardiol 1992; 35: 387-395.

30 Duranay $M$, Kanbay $M$, Akay $H$, Unverdi S, Sürer $H$, Altay $M$ et al. Nebivolol improves renal function in patients who underwent angioplasty due to renal artery stenosis: a pilot study. Nephron Clin Pract 2010; 114: c213-c217.

31 Günebakmaz O, Kaya MG, Koc F, Akpek M, Kasapkara A, Inanc MT et al. Does nebivolol prevent contrast-induced nephropathy in humans? Clin Cardiol 2012; 35: 250-254.

32 Ponikowski P, Voors AA, Anker SD, Bueno H, Cleland JG, Coats AJ et al. ESC guidelines for the diagnosis and treatment of acute and chronic heart failure: The Task Force for the diagnosis and treatment of acute and chronic heart failure of the European Society of Cardiology (ESC) developed with the special contribution. Eur J Heart Fail 2016; 18: 891-975.

33 González-García S, Hernández-Díaz Z, Quevedo-Sotolongo L, Peña-Sánchez M, Pino-Peña $\mathrm{Y}$, Fernández-Carriera $\mathrm{R}$ et al. Resistive cerebral blood flow as a potential marker of subclinical brain damage in essential hypertension. World $J$ CVD 2014; 4: 169-178.

34 Cohuet G, Struijker-Boudier H. Mechanisms of target organ damage caused by hypertension: therapeutic potential. Pharmacol Ther 2006; 111: 81-98.

35 González-Quevedo A, García SG, Concepción OF, Freixas RS, Sotolongo LQ, Sánchez MP et al. Demonstration of subclinical organ damage to the central nervous system in essential hypertension. In: Gonzalez-Quevedo A (eds). Brain Damage-Bridging Between Basic Research and Clinics. InTech: Rijeka, Croatia, 2012, pp 79-98.

36 Ohtsuki T, Matsumoto M, Suzuki K, Taniguchi N, Kamada T. Mitochondrial lipid peroxidation and superoxide dismutase in rat hypertensive target organs. Am J Physiol 1995; 268: H1418-H1421.

(2) This work is licensed under a Creative Commons AttributionBY NC SA NonCommercial-ShareAlike 4.0 International License. The images or other third party material in this article are included in the article's Creative Commons license, unless indicated otherwise in the credit line; if the material is not included under the Creative Commons license, users will need to obtain permission from the license holder to reproduce the material. To view a copy of this license, visit http:// creativecommons.org/licenses/by-nc-sa/4.0/

(c) The Author(s) 2017 